\title{
Maxime Quijoux, Adieux au patronat. Lutte et gestion ouvrières dans une usine reprise en coopérative
}

Vulaines-sur-Seine, Éditions du Croquant, coll. « Dynamiques socioéconomiques ", 2018

Anne-Catherine Wagner

\section{(2) OpenEdition}

Journals

Édition électronique

URL : https://journals.openedition.org/travailemploi/9939

DOI : $10.4000 /$ travailemploi.9939

ISSN : 1775-416X

Éditeur

DARES - Ministère du Travail

Édition imprimée

Date de publication : 1 mars 2020

Pagination : 124-127

ISSN : 0224-4365

Référence électronique

Anne-Catherine Wagner, « Maxime Quijoux, Adieux au patronat. Lutte et gestion ouvrières dans une usine reprise en coopérative », Travail et Emploi [En ligne], 161 | 2020, mis en ligne le 01 mars 2021, consulté le 27 septembre 2021. URL : http://journals.openedition.org/travailemploi/9939 ; DOI : https://doi.org/ 10.4000/travailemploi.9939 


\title{
Adieux au patronat. Lutte et gestion ouvrières dans une usine reprise en coopérative
}

\author{
Maxime Quijoux
}

Vulaines-sur-Seine, Éditions du Croquant, coll. « Dynamiques socio-économiques », 2018, 318 p.

Lu par Anne-Catherine Wagner ${ }^{*}$

L'ouvrage du sociologue Maxime Quijoux relate une expérience sociale a priori improbable. Des salariés d'une imprimerie menacée de fermeture parviennent en 2012, sous l'impulsion de la Confédération générale du travail (CGT), à reprendre leur entreprise sous forme de société coopérative et participative (Scop) : ils sauvent 80 emplois, relancent l'activité et mettent en place des formes alternatives de gestion. En revenant sur les différentes étapes de cette reprise d'entreprise, l'auteur présente une dimension méconnue de l'action syndicale et montre comment la «professionnalisation » des syndicalistes peut constituer une arme de résistance salariale. L'analyse prend appui sur une enquête au long cours menée à l'imprimerie Hélio-Corbeil, spécialisée dans la technique de l'héliogravure et située à Corbeil-Essonnes. M. Quijoux y a passé 22 semaines comme stagiaire entre octobre 2012 et avril 2013; il y a conduit des entretiens, des observations, et dépouillé des archives syndicales et d'entreprise.

La première partie revient sur les conditions sociales qui ont amené un groupe d'ouvriers à reprendre leur imprimerie en Scop. Dans ce secteur d'activité où le syndicalisme catégoriel est puissant et les conventions collectives avantageuses, l'entreprise rachetée par Hachette à la fin des années 1970 offre des conditions de promotions et de rémunérations particulièrement favorables. Tout cela concourt à un sentiment d'appartenance fort, qui tient aussi au prestige d'un type d'impression qui requiert des savoir-faire spécifiques. Les salariés de cette imprimerie se caractérisent ainsi par un ensemble de propriétés qui les situent vers le haut de l'espace des classes populaires contemporaines. La vie à l'usine est marquée par de fortes sociabilités d'atelier et des habitudes de résistance à la hiérarchie. La CGT y a toujours été bien implantée, combinant combativité et expertise économique et technique. Les délégués s'apparentent ainsi à des «intellectuels d'industrie » (p. 51): leurs positions relativement élevées dans la distribution sociale de l'entreprise et leurs capitaux militants, souvent façonnés par une socialisation familiale et militante communiste, les prédisposent à se sentir légitimes pour revendiquer un contrôle de la gestion de l'entreprise.

* Université Paris 1 Panthéon Sorbonne, Centre européen de sociologie et de science politique (CESSP). 
Ces dispositions gestionnaires s'affirment dans les années 2000, quand s'ouvre une période de restructuration, liée à la fois à la crise de l'industrie graphique et aux stratégies financières des nouveaux propriétaires. Le projet industriel devient la pierre angulaire du combat syndical. De nouvelles alliances sont trouvées, d'une part, avec Serge Dassault, maire de droite de Corbeil-Essonnes, qui trouve son intérêt à soutenir les industries emblématiques de la région, d'autre part, avec les pouvoirs publics. En dépit d'un revers syndical sans précédent quand, en 2006, lors d'un plan social, de nombreux élus CGT partent avec des primes, le syndicat se redresse grâce à de nouvelles recrues et est en position de porter le projet de reprise six ans plus tard.

La deuxième partie de l'ouvrage pose la question du type de gouvernance coopérative mise en place dans la Scop d'Hélio-Corbeil. À rebours de l'analyse des sciences de gestion qui présuppose une inclination «naturelle » des salariés à participer à la vie de l'entreprise, l'auteur met en lumière les tensions autour de différentes manières de s'impliquer dans le redressement de l'imprimerie. Les nouvelles hiérarchies ne subvertissent pas totalement les principes de divisions qui structuraient l'entreprise et le syndicat: les cadres conservent leurs positions dominantes, le délégué syndical devient le nouveau président directeur général (PDG) de la Scop et toute l'équipe syndicale entre au conseil d'administration, cette promotion collective n'excluant pas des désaccords sur les priorités de la Scop. Les autres salariés, moins pourvus en compétences techniques, politiques et sociales, se retrouvent en situation dominée. L'auteur analyse des formes diffuses de régulation démocratique entre ces deux pôles: ainsi, alors que les salariés ne s'opposent pas directement aux nouveaux dirigeants, au moment de l'assemblée générale par exemple, au sein de l'atelier, ils n'hésitent pas à faire pression sur leurs collègues membres du conseil d'administration.

L'enquête met en évidence le décalage entre la conception syndicale de la coopérative et celle promue par le mouvement coopératif qui cherche à faire valoir, via une action de formation dans l'imprimerie, une conception plus verticale de la démocratie en entreprise. Les reprises d'entreprises « en difficulté » ne sont pas un sujet d'intérêt prioritaire pour la Confédération générale des Scop, qui préfère mettre l'accent sur les coopératives les plus performantes dans sa communication. De leur côté, les dirigeants d'Hélio-Corbeil sont rétifs vis-à-vis de la figure entrepreneuriale mise en avant par le mouvement Scop. L'imprimerie de Corbeil-Essonnes est ainsi l'une des rares coopératives françaises évoluant hors de son giron.

La troisième partie pose la question de la «gestion cégétiste » d'une entreprise. L'auteur souligne les conditions défavorables dans lesquelles le syndicat reprend l'entreprise : vétusté des machines, nombreux postes laissés vacants, tensions internes. Dans ce contexte, la nouvelle direction met en œuvre une politique de relance productive avec embauches et investissements, mobilise toute une palette de ressources syndicales (soutien de la fédération, réseau de solidarité interentreprises) et brouille - en partie - la frontière entre décideurs et exécutants. Cette gestion est marquée par l'informalité des pratiques et une hybridation de conduites militantes et de fonctions dirigeantes. La confrontation avec la concurrence ou les clients hostiles ont pourtant 
des effets sur les dispositions économiques des dirigeants : l'ancien délégué syndical adhère à l'union patronale de la branche, ce qui peut aussi s'analyser comme un effet de la plasticité du statut coopératif qui donne des leviers d'action nouveaux. Qu'un ancien délégué syndical devienne PDG n'affaiblit pas la position de la CGT, dont le nombre d'adhérents augmente au contraire dans l'imprimerie. En revanche, la promotion de syndicalistes au sein de la hiérarchie de l'entreprise a pour conséquence de les éloigner des salariés d'exécution et de les rapprocher des cadres, évolution qui se traduit par une élévation sociale du recrutement des administrateurs par rapport à la période suivant la reprise. L'étude se termine avec un plaidoyer pour un «syndicalisme de combat économique » qui pourrait régénérer l'action syndicale et donner une dynamique nouvelle au secteur de l'économie sociale et solidaire.

Cet ouvrage est précieux par l'éclairage qu'il apporte sur un type d'entreprise encore peu exploré par les sociologues. La posture de l'auteur lui permet de proposer une fine analyse, de l'intérieur, du fonctionnement au quotidien de cette imprimerie particulière. En évitant de faire l'apologie d'un «miracle social » ou au contraire de dénoncer de nouvelles formes de domination, il trouve, comme dans sa précédente enquête sur les usines récupérées en Argentine ${ }^{1}$, le ton juste pour analyser les obstacles, les contradictions et les potentialités de ces situations où des ouvriers sont amenés à gérer leur entreprise. L'analyse s'appuie sur un riche matériau ethnographique, et les nombreux portraits de salariés et de militants donnent bien à comprendre la diversité des points de vue et des positions dans la coopérative. La temporalité de l'enquête est l'un de ses points forts: la longue présence de l'auteur sur son terrain et son souci de relier les événements présents au passé de l'imprimerie se révèlent particulièrement heuristiques.

Les efforts déployés par l'auteur pour être nuancé et honnête dans sa restitution des faits et dans ses analyses peuvent quelquefois provoquer des frustrations chez le lecteur, confronté à la diversité des lectures qui peuvent être faites des conflits ou des rapports décrits: la coopérative ne fait pas disparaître les inégalités mais brouille les anciennes frontières; la promotion collective des syndicalistes les éloigne de la base, qui garde néanmoins des moyens de pression; certains mystères ne sont pas totalement élucidés, comme celui de l'effet (ou de l'absence d'effet?) de la trahison d'une grande partie des élus CGT partis avec des primes quelques années avant la mise en place de la coopérative.

Que l'imprimerie étudiée - qui a récemment mis fin à ses activités - soit très spécifique (y compris parmi les Scop) met précisément en lumière le fait que ces reprises d'entreprises ne sont possibles que dans des circonstances particulières. Cette spécificité pose néanmoins la question des conditions d'une montée en généralité. Cette monographie passionnante suscite le désir d'une plus grande mise en perspective, notamment sur les transformations du rapport du syndicalisme à l'autogestion, évoquées par l'auteur en conclusion. Comment de telles expériences produisent-elles leurs 
effets sur les syndicats, à la CGT bien sûr, mais aussi au sein des autres syndicats, absents de l'imprimerie étudiée ? Dans quelle mesure l'intervention gestionnaire estelle source de nouveaux clivages syndicaux ou militants? Cette riche enquête ouvre ainsi toute une série de pistes et de questions pour repenser les rapports ambivalents à la participation dans l'entreprise. 